\title{
Sabin Vaccine in Poliomyelitis Eradication: Achievements and Risks
}

\author{
Omar A. Nafi* and Bashar Ramadan \\ Department of Pediatrics, Faculty of Medicine, Mutah University, Al Karak, Jordan.
}

\begin{abstract}
Poliomyelitis eradication using the oral polio vaccine (OPV), also known as the Sabin vaccine, has been a major medical achievement led by the World Health Organization (WHO) and various countries. The OPV has been administered over 10 billion times to three billion children and has prevented over 13 million polio cases. With the more recent appearance of OPV-related complications, especially the vaccine-derived poliovirus (VDPV) and vaccine-associated polio paralysis, it is important to reconsider the role of this vaccine in polio eradication. Since 2014, the number of VDPV cases has exceeded the number of wild polio virus cases. Given that OPV is the only source of VDPV, an established phased plan to withdraw OPV from use and switch to an inactivated polio vaccine (IPV) was determined. Therefore, countries that still use the OPV in their national immunization programs need to develop adequate plans for supplying IPV in an effective and affordable manner. IPV provides protection against polio, but is insufficient for protection against poliovirus circulation when administered alone. Genetically engineered stabilized, live vaccines are being developed and guarantee the profit of Sabin OPV without the risk.
\end{abstract}

Keywords: Oral polio vaccine (OPV), wild polio virus, vaccine-associated polio paralysis (VAPP), vaccine-derived poliovirus (VDPV).

\footnotetext{
*Correspondence: onafi2000@yahoo.com,omarnafi@mutah.edu; +962 796548783
}

(Received: 15 January 2019; accepted: 27 February 2019)

Citation: Omar A. Nafi and Bashar Ramadan, Sabin Vaccine in Poliomyelitis Eradication: Achievements and Risks, J Pure Appl Microbiol., 2019; 13(1):413-418 doi: 10.22207/JPAM.13.1.45

C The Author(s) 2019. Open Access. This article is distributed under the terms of the Creative Commons Attribution 4.0 International License which permits unrestricted use, sharing, distribution, and reproduction in any medium, provided you give appropriate credit to the original author(s) and the source, provide a link to the Creative Commons license, and indicate if changes were made. 


\section{INTRODUCTION}

After decades of being administered to several billion children globally as well as being credited as primarily responsible for the eradication of poliomyelitis, the Sabin vaccine is now being considered for withdrawal from use. The history of the vaccine, its impact on human health, the rationale for its withdrawal, the alternative use of inactivated polio vaccine (IPV), and associated risks of this substitution are discussed in this short communication.

\section{History of Sabin vaccine}

Albert Sabin published an article in 1960 describing the effects of the new trivalent oral polio vaccine (tOPV) when administered to approximately 26,000 children in Cuba ${ }^{1}$. The new vaccine was licensed in the USA after 1961 due to its superior level of antibody production and sufficiently rare neurotropic effects on monkeys. Cuba was the first country to conduct a polio vaccination campaign on a national level ${ }^{2}$. Sabin provided his investigational data and strains of polio to Chumakov, a Soviet microbiologist, in 1956. Chumakov later started organizing the production of the OPV for his state, and only some million children in the Soviet Union were administered this vaccine by 1959 . The success of Chumakov's efforts later led to the licensing of the OPV ${ }^{3}$.

The OPV consists of three strains of live attenuated poliovirus. This vaccine was easier to administer compared to its injectable counterpart and resulted in a so-called herd effect ${ }^{4}$. It also offered longer-lasting immune protection, which included systemic, humoral, cellular, and mucosal immunity (OPV was the superior choice over IPV for global eradication for three main reasons; lower production cost, ease of administration and its ability to induce mucosal immunity)

OPV was preferred over IPV as the vaccine for global eradication due to its capacity to induce mucosal immunity, its lesser production price, and easiness of administration.

Sabin donate his vaccine strains in 1972 to the World Health Organization (WHO) to allow the vaccine to reach more people in developing countries. As a result of this donation, the percentage of children worldwide who received the full course of OPV increased from $5 \%$ to $80 \%$ in only two decades ${ }^{6}$.

\section{Eradication of poliomyelitis}

A proposal to eradicate polio was made in 1988 by the WHO. A strategic plan outlined the requirements for achieving this goal, which included certification-indicating eradication for regions, as well as two phases: an OPV ending phase and a post-OPV phase. The plan mandated vaccination coverage of more than $80 \%$ amongst children, with additional vaccine doses provided during national vaccine days. One dose of the vaccine leads to $50 \%$ of children developing immunity against the strains; three doses lead to $95 \%$ or more. Several surveillance systems for polio were also set in place, as were containment protocols.

\section{Wild poliovirus}

Of the three strains of wild poliovirus, the type 2 strain was last reported to cause poliomyelitis in 1999 and was formally certified as eradicated since 2015 . The type 3 strain was last reported in 2012 in Nigeria. Since 2012, every case of poliomyelitis has been attributed to the type 1 strain and has occurred in one of only three countries: Nigeria, Pakistan, and Afghanistan? There has been steady progress in both Pakistan and Afghanistan. In 2016, Pakistan reported 20 cases of poliomyelitis; in 2017, there were only 5 reported cases. Similarly, in Afghanistan, a total of 13 cases were reported in 2016, with only 6 reported cases in 2017 (Circulating vaccinederived poliovirus type $2-$ Nigeria). There are three countries in the globe with current wild poliovirus spread, namely, Nigeria, Afghanistan, and Pakistan. These countries are also affected by circulating vaccine-derived poliovirus type 2 (cVDPV2) outbreaks.

\section{Achievements of oral polio vaccine}

The OPV has been administered over 10 billion times to three billion children and has prevented over 13 million cases of polio and has led to a $99 \%$ reduction in polio incidence 8 .

In 1988,350,000 cases of paralytic polio cases were reported worldwide in 125 endemic countries at a moment in time when the World Health congregation was trying to eradicate polio?.

In 2018, only 29 cases of wild polio paralysis have been reported worldwide; these cases have all been reported from three endemic countries, namely, Afghanistan, Nigeria, and Pakistan (Fig. 1). 


\section{Risks of oral polio vaccine}

Vaccine-associated paralytic poliomyelitis (VAPP)

Although OPV contains weakened polio strains, these strains can still result in poliomyelitis in one per 2.7 million recipients ${ }^{10}$. This is known as vaccine-associated paralytic poliomyelitis (VAPP), which is caused by a genetically modified virus that is formed in the small intestine after the administration of the vaccine. VAPP not only affects those who have been vaccinated, but also the unvaccinated or non-immune close contacts. In children with healthy immune systems, the incidence of VAPP is an estimated one in 750,000 doses; this rate is one in 6.9 million for subsequent doses ${ }^{11}$. Even though the type 3 strain is the most widespread cause of VAPP ${ }^{12}$ the type 2 strain has been associated with poliomyelitis in the close contacts of those who have received the vaccine.

\section{Vaccine-derived poliovirus (VDPV)}

Vaccine-derived polioviruses (VDPVs) are uncommon strains of poliovirus that have genetically mutated from the strains enclosed in the OPV.

The live attenuated vaccine-virus may no longer be similar to the original vaccinevirus as it has genetically altered throughout replication in the intestine ${ }^{13}$. If the virus circulates for 12-18 months, an individual may reacquire neurovirulence. These viruses are called circulating vaccine-derived polioviruses (cVDPV). The survival of these viruses is inversely comparative to the population immunity; the more they replicate, the more they transform, and exchange genetic material with other entero viruses as they spread through the population ${ }^{14}$.

The low rate of vaccination coverage is the main problem for development of vaccine derived poliovirus, more than the vaccine itself. If a community is entirely immunized, they will be protected against both the vaccine-derived and wild polioviruses ${ }^{15}$.

Vaccine derived polioviruses have recently started to appear worldwide and are genetically different ${ }^{16,17,18,19}$ from previous strains of polioviruses.

\section{Types of VDPVs}

There are three types of VDPVs: circulating VDPV, immunodeficiency-related VDPV, and ambiguous VDPV.

\section{Circulating cVDPV}

Outbreaks of circulating vaccine-derived poliovirus are uncommon. During the period 2000-2011 - a time in when over 10 billion doses of OPV were administered worldwide - 20 cVDPV outbreaks registered, leading to 580 polio cases. In the similar time, without vaccination with OPV, around six million children would have been paralyzed by poliovirus.

A total of 108 cases of cVDPV2 cases were reported from 2016 to 2018 . These cases were related to 8 outbreaks in 5 countries (Somalia,

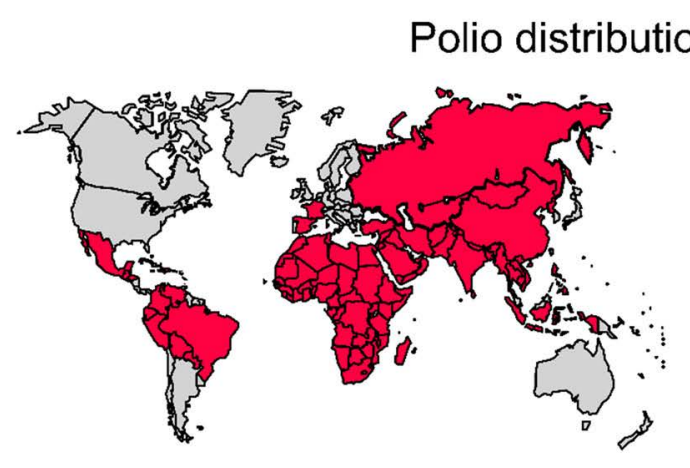

1988

- 350,000 cases

- 125 endemic countries

- World Health Assembly resolved to eradicate polio

\section{past and present}

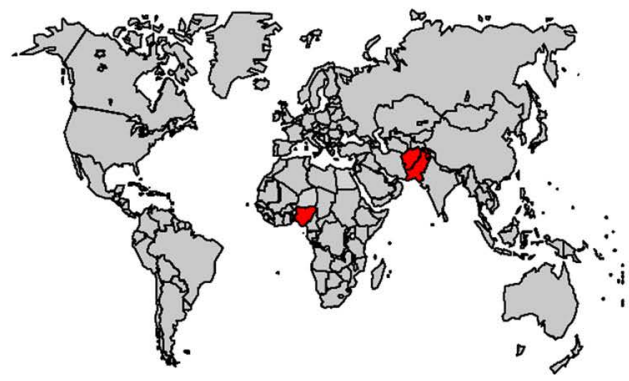

2018

- 29 cases reported (total 2018)

- 3 endemic countries

Fig. 1. Map of polio distribution, past and present 
Nigeria, Congo, Pakistan, and Syria). Most of the cases were reported in Syria ${ }^{20}$.

\section{Immunodeficiency-related VDPV}

A few cases have been reported where lengthened reproduction of VDPVs have been noted in people with severe immune deficiency. The lack of an effective immune response leads to the inability of clearing the intestinal infection, which is normally supposed to be cleared within 6-8 weeks. Therefore, they continue to expel immunodeficiency-related VDPVs (iVDPVs) for expanded periods.

Studies from 25 countries (49 published manuscripts) from January 1960 to November 2012 reported 68 iVDPV cases. The VDPV represent a challenge to the worldwide polio eradication as it may serve as a source of the virus even after the eradication of wild-type ${ }^{21}$.

\section{Ambiguous VDPV}

Ambiguous VDPV is understood to be VDPV isolated from persons without identified immunodeficiency. A VDPV isolate is considered "ambiguous" when further investigations have excluded that it is part of an continuing chain of transmission, i.e. a cVDPV, or derived from an iVDPV22.

\section{Management of vaccine-derived polioviruses}

The current recommendation is to vaccinate every child several times with OPV to end polio spread, in spite of whether the virus is wild or vaccine-derived. VDPVs appear to be not as much transmissible as the wild poliovirus, and outbreaks of VDPV are typically self-limiting or quickly blocked with 2-3 rounds of highquality supplementary immunization activities. Nowadays, the quantity of polio cases due to OPV is greater than that related to the wild virus.

Provided that OPV is used, VDPVs creates a threat of causing poliomyelitis in vulnerable individuals, which makes eradication difficult. Inactivated poliovirus vaccine (IPV) solves this problem since VDPV would not be able to form from an inactivated virus. However, economic and logistical barriers make switching to IPV difficult ${ }^{23}$. What will occur following the removal of oral polio vaccine and the use of IPV only?

Several developed countries, which have better sanitation and smaller family sizes, have preserved polio elimination through the use of IPV $24,25,26$. This is because IPV alone does not have the risk of spreading the virus in the stool.

Although IPV has been included in routine vaccination around the world between 2014 and $2016^{27}$, it is anticipated that IPV alone will not be sufficient to protect against the virus in all settings. Improvements in sanitation, as well as better IPV supplies ${ }^{25}$ will also be necessary.

Currently, genetically engineered live vaccines are being developed, this will hopefully maintain the benefits of the Sabin OPV without the associated risks ${ }^{28,29,30}$, and adjuvant IPV may supply a complementary route to a new effective vaccine ${ }^{31}$. We believe these new types of vaccines will be necessary to insure polio eradication.

Mass immunization with OPV remains the most efficient intervention to eliminate poliovirus transmission ${ }^{32}$, because in spite of the challenges mentioned, Sabin vaccination is clearly preferable to natural infection by wild polio virus or cVDP.

\section{CONCLUSION}

Polio is an extremely communicable viral disease that can spread quickly through personto-person contact leading to everlasting paralysis. At present three types of wild poliovirus but only type 1 still in circulation nowadays. OPV vaccine is inexpensive and efficient at reducing polio spread in developing countries but carries a danger of VAPP and VDPV; therefore, all use of OPV have to end for the humanity to be entirely polio-free. IPV is being introduced to afford defense against all three serotypes, at the same time as OPV is being phased out.

Therefore, the countries that still use this vaccine (OPV) in their national immunization programs need to develop adequate plans for supplying IPV in an effective and affordable manner. The IPV provides protection against polio but alone is not enough to defend against poliovirus transmission.

To secure polio eradication for the future, genetically stabilized, engineered live vaccines are in progress and pledge the profit of the Sabin OPV with no risks.

The WHO has stated that poliomyelitis (polio) is on the way to turn into the next human disease forever to be eradicated as only 29 cases of wild poliovirus have been detected thus far in 2018 in Afghanistan, Nigeria, and Pakistan, the three lasting endemic countries. 
At the same time as a $99.9 \%$ decrease in polio cases globally, the World Health Organization (WHO) said tackling the remaining $0.01 \%$ of polio cases has demonstrated to be difficult and warned that immunization efforts have to continue.

In spite of the risks fully discussed in this article, Sabin OPV immunization is for all time preferable to get the disease by wild polio virus or CVDPV. Mass immunization with OPV remains therefore the most efficient intervention to stop poliovirus transmission.

\section{ACKNOWLEDGMENTS}

None

\section{CONFLICT OF INTEREST}

The authors declare that there are no conflict of interest.

\section{REFERENCES}

1. Sabin AB, Ramos-Alvarez M, Alvarez-Amezquita J, Pelon W, Michaels RH, Spigland I, Koch MA, Barnes JM, Rhim JS. Live, orally given poliovirus vaccine. Effects of rapid mass immunization on population under conditions of massive enteric infection with other viruses. JAMA, 1984; 251: 2988-2993.

2. Hinman AR. Mass vaccination against polio. JAMA, 1984; 251: 2994-2996.

3. Agol VI and Drozdov SG. Russian contribution to OPV. Biologicals, 1993; 21: 321-325.

4. Nathanson Nand Kew OM. From emergence to eradication: The epidemiology of poliomyelitis deconstructed. Am. J. Epidemiolog., 2010; 172: 1213-1229.

5. Plotkin $S A$ and Vidor E. Poliovirus vaccine-inactivated. In: Plotkin SA, Orenstein WA, and Offit PA (Eds.), Vaccines 5. Elsevier Inc., Philadelphia, 2008; 605-629.

6. Baicus A. History of polio vaccination. World J. Virol., 2012; 1: 108-114.

7. Mehndiratta MM, Mehndiratta P, Pande R. Poliomyelitis-Historical Facts, Epidemiology, and Current Challenges in Eradication. Neuro-hospitalist, 2014; 4: 223-229.

8. World Health Organization, "What is vaccinederivedpolio?", 2017 https://www.who.int/features/ qa/64/en/(Nov.12, 2018)

9. Khan F, Datta SD, Quddus A, Vertefeuille JF, Burns CC, Jorba J, Wassilak SGF. Progress toward polio eradication-worldwide, January 2016-March 2018. MMWR Morb. Mortal. Wkly. Rep., 2018; 67: 524-528.

10. Prevots DR, Sutter RW, Strebel PM, Weibel RE, Cochi SL. Completeness of reporting for paralytic poliomyelitis, United States, 1980 through 1991: implications for estimating the risk of vaccine-associated disease. ArchPediatrAdolesc Med, 1994; 148: 479-485.

11. Strebel PM, Sutter RW, Cochi SL, Biellik RJ, Brink EW. Epidemiology of poliomyelitis in the United States one decade after the last reported case of indigenous wild virus-associated disease. Clin. Infect. Dis., 1992; 14: 568-579.

12. Platt LR, Estovariz CF, Sutter RW. Vaccine-associated paralytic poliomyelitis: A review of the epidemiology and estimation of the global burden. J. Infect. Dis., 2014; 210: S380-S389.

13. Fact sheet on vaccine-derived poliovirus published by the Global Polio Eradication Initiative 2017 (PDF) (https://www.who.int/features/qa/64/en/). Dec.18.2018.

14. Jorba J, Diop OM, Iber J,Henderson E, Sutter RW, Wassilak SGF, Burns CC. Update on vaccine-derived polioviruses - worldwide, January 2016-June 2017. MMWR Morb. Mortal Wkly Rep., 2017; 66: 1185-1191.

15. World Health Organization, Regional Office for Africa. "Circulating vaccine-derived poliovirus type 2 (VDPV2) Key messages". https://afro.who.-int/node/9733 (June 6, 2018).

16. Centers for Disease Control and Prevention. "Epidemiology and Prevention of Vaccine-Preventable Disease."https://www.cdc.gov/vaccines/pubs/ pinkbook/polio.html (Nov.12, 2018).

17. Kew OM, SutterRW, Nottay B, McDonough MJ, Prevots DR, Quick L, Pallansch MA.Prolonged replication of a type 1 vaccine-derived poliovirus in an immunodeficient patient. J. Clin. Microbiol., 1998; 36: 2893-2899.

18. Kew OM, Sutter RW, deGourville EM,Dowdle WR, Pallansch MA. Vaccine-derived polio viruses and the endgame strategy for global polio eradi-cation. Annu. Rev. Microbiol., 2005; 59: 587-635.

19. Li L, Ivanova O, TrikiH, Tiongco-Recto M, Da Silva R, Shahmahmoodi S, Sazzad HM, Mach O, Kahn AL, Sutter RW. Poliovirus excretion among persons with primary immune deficiency disorders: Summary of a seven-country study series. J. Infect. Dis., 2014; 210: S368-S372.

20. Jorba J, Diop OM, Iber J. Update on vaccine-derived polioviruses - worldwide January-June 2018. MMWR Morb. Mortal Wkly Rep., 2017; 67: 1189-1194.

21. Guo J, Bolivar-Wagers S, Srinivas N, Holubar M, Maldonado Y. Immunodeficiency-related vaccinederived poliovirus (iVDPV) cases: a systematic review and implications for polio eradication. Vaccine, 2015; 33: $1235-1242$.

22. (http://polioeradication.org/wp-content/ uploads/2016/09/Reporting-and-Classification-ofVDPVs_Aug2016_EN.pdf)

23. Minor P.Vaccine-derived poliovirus (VDPV): Impact on poliomyelitis eradication. Vaccine, 2009; 27: 26492652.

24. Alexander LN, Seward JF, Santibanez TA, Pallansch MA, Kew OM, Prevots DR. Vaccine policy changes and epidemiology of poliomyelitis in the United States. JAMA, 2004; 292: 1696-1701.

25. HamptonLM, Farrell M, Ramirez-Gonzalez A, Menning $\mathrm{L}$, Shendale S, Lewis I. Cessation of trivalent oral poliovirus vaccine and introduction of inactivated poliovirus vaccine -worldwide, 2016. MMWRMorb Mortal Wkly Rep., 2016; 65: 934-938.

26. Pebody R. Polio vaccination in Europe: the shift from 
OPV to IPV use. Euro. Surveill., 2004; 8: 2529.

27. Sutter RW, Platt L, Mach O, Jafari H, Aylward RB.The new polio eradication end game: Rationale and supporting evidence. J. Infect. Dis., 2014; 210(suppl 1): S434-\$438.

28. Bandyopadhyay AS, Garon J, Seib K, Orenstein WA. Polio vaccination: past, present and future. Future Microbiol, 2015; 10: 791-808.

29. Macadam AJ, Ferguson G, Stone DM, Meredith J, Knowlson S, Auda G. Rational design of genetically stable, live-attenuated poliovirus vaccines of all three serotypes: relevance to poliomyelitis eradication. $J$.
Virol., 2006; 80: 8653-8663.

30. Lauring AS, Jones JO, Andino R.. Rationalizing the development of live attenuated virus vaccines. Nature Biotech., 2010; 28: 573-579.

31. Norton EB, Bauer DL, Weldon WC, Oberste MS, Lawson LB, Clements JD. The novel adjuvant dmLT promotes dose sparing, mucosal immunity and longevity of antibody responses to the inactivated polio vaccine in a murine model. Vaccine, 2015; 33: 1909-1915.

32. Sutter RW and Maher $\mathrm{C}$. Mass vaccination campaigns for polio eradication: an essential strategy for success. Curr. Top Microbiol. Immunol., 2006; 304: 195-220. 